\title{
Emerging Trends in Biomaterials Research
}

It is our great pleasure to introduce the June 2016 special issue of the Annals of Biomedical Engineering. Across the broad spectrum of our field, biomaterials permeate nearly everything we do. Advances in biomaterials technology often have a ripple effect, and collectively, these advances elevate our entire field. Therefore, this special issue is focused on emerging trends in biomaterials ranging from the nanoscale to the macroscale, for a wide range of biomedical applications, including therapeutic delivery, immunotherapy, bioimaging, and regenerative engineering.

Traditionally, single component biomaterials have suffered from serious limitations due to limited control over biophysical and biochemical characteristics, hampering their utility for biomedical applications. To overcome these limitations, a range of advanced biomaterials are designed with multiple functionalities to guide cell behavior. This special issue emphasizes the design and development of the next generation of smart and responsive biomaterials to address these challenges. The original research articles capture the growing trend of functional biomaterials, and the review articles provide a critical evaluation of emerging trends in designing the next generation of biomaterials.

Engineering new biomaterials from natural sources such as marine sponges and decellularized tissues (cartilage) (Boccaccini, Detamore) are used to mimic biophysical and biochemical characteristics of native tissues. Other approaches include engineering natural polymers such as gelatin and alginate to co-encapsulate cells and therapeutics for guiding cellular functions (Mikos, Moshaverinia). Cell-instructive biomaterials are designed by incorporating biochemical clues to promote, augment, and facilitate regeneration of the damaged tissues (Burdick, Gu, Garcia, Bryant). These studies highlight that controlling cell-material interactions is necessary for modulating cellular functions.

"Smart" biomaterials are another emerging class of materials that respond to multiple external stimuli. For example, biomaterials responding to $\mathrm{pH}$ or cell-secreted enzymes can be used for on-demand and local delivery of therapeutics to regulate cell responses (Peppas, Segura). By utilizing the physicochemical characteristics of smart biomaterial, effective delivery of labile biomolecules is proposed for immunomodulation and long-term disease management (Mitragotri, Tasciotti). Smart and responsive biomaterials with multiple functionalities are promising biomaterials for a range of biomedical applications.

Nanoengineered biomaterials present significant opportunities to design materials with custom properties. By incorporating nanoparticles within a polymeric network, biomaterials with tailored functionality have been developed. A range of two- and three-dimensional nanomaterials have been shown to physically or chemically interact with the polymers, yielding new characteristics of the nanoengineered network (Sitharaman). Nanocomposite biomaterials with tailored functionality have opened up new possibilities for modulating cellular behavior for tissue engineering, localized drug delivery, and osteoarthritis (Sant, Zhang, Singh).

Advanced manufacturing technologies such as microfabrication and 3D printing enable mimicry of complex tissue architectures and can provide the essential cellular microenvironment to guide the formation of functional tissues. For example, microscale geometric patterning of ECM

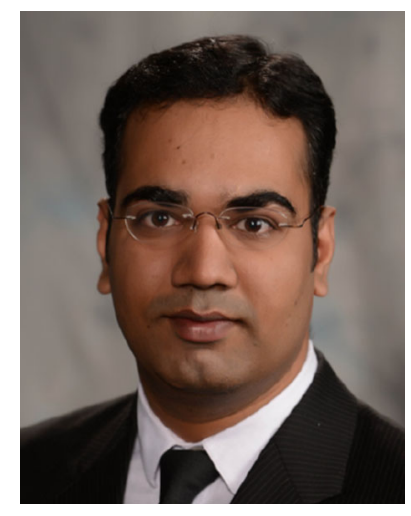

Akhilesh K. Gaharwar

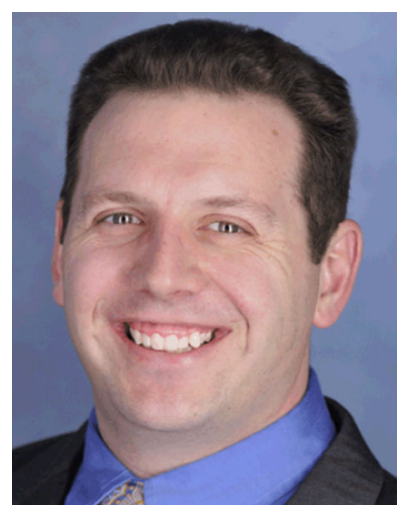

Michael S. Detamore

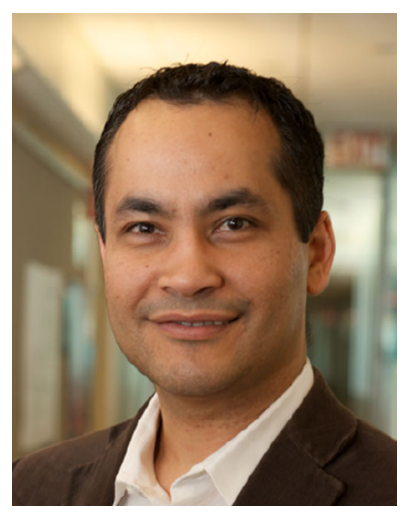

Ali Khademhosseini proteins can be leveraged to control cell alignment and differentiation (Feinberg). These microfabricated structures may be employed for 
in vitro applications in toxicity screening, disease modeling and drug discovery. Another emerging approach to pattern and guide cell behavior is $3 \mathrm{D}$ printing. A vital aspect and bottleneck to the design and implementation of a bioprinting system is a lack of suitable bioinks that are printable and can guide cell functions (Gaharwar). To address these challenges, a range of advanced bioink formulations is designed including multicomponent systems, interpenetrating networks, nanoengineered bioinks and supramolecular networks.

Collectively, the results from the body of work presented in this special issue are very exciting, representing several key advances in harnessing the power of biomaterials to revolutionize healthcare.
AkHiLesh K. Gaharwar

Texas A\&M University, College Station, TX, USA

Electronic mail: gaharwar@tamu.edu

Michael S. Detamore

University of Kansas, Lawrence,

KS, USA

Electronic mail: detamore@ku.edu

Ali Khademhosseini

Harvard University, Cambridge, MA, USA

Electronic mail: alik@bwh.harvard.edu 